\title{
Prevention of airway hyperresponsiveness induced by left ventricular dysfunction in rats
}

\author{
Ferenc Petak ${ }^{1 *}$, Gergely Albu², Eniko Lele ${ }^{2}$, Maurice Beghetti ${ }^{3}$ and Walid Habre ${ }^{4}$
}

\begin{abstract}
Background: The effectiveness of strategies for treatment of the altered static lung volume and against the development of bronchial hyperreactivity (BHR) following a left ventricular dysfunction (LVD) induced by myocardial ischaemia was investigated in a rat model of sustained postcapillary pulmonary hypertension.

Methods: Airway resistance (Raw) was identified from the respiratory system input impedance (Zrs) in four groups of rats. End-expiratory lung volume (EELV) was determined plethysmographically, and Zrs was measured under baseline conditions and following iv infusions of 2,6 or $18 \mu \mathrm{g} / \mathrm{kg} / \mathrm{min}$ methacholine. Sham surgery was performed in the rats in Group C, while the left interventricular coronary artery was ligated and Zrs and its changes following identical methacholine challenges were reassessed in the same rats 8 weeks later, during which no treatment was applied (Group I), or the animals were treated daily with a combination of an angiotensin enzyme converter inhibitor and a diuretic (enalapril and furosemide, Group IE), or a calcium channel blocker (diltiazem, Group ID). The equivalent dose of methacholine causing a $100 \%$ increase in Raw $\left(\mathrm{ED}_{50}\right)$ was determined in each group. Diastolic pulmonary arterial pressure ( $\mathrm{Pap}_{\mathrm{D}}$ ) was assessed by introducing a catheter into the pulmonary artery.

Results: The sustained presence of a LVD increased $P a p_{D}$ in all groups of rats, with variable but significant elevations in Groups I $(p=0.004)$, ID ( $p=0.013)$ and IE $(p=0.006)$. A LVD for 8 weeks induced no changes in baseline Raw but elevated the EELV independently of the treatments. In Group I, BHR consistently developed following the $L V D$, with a significant decrease in $\mathrm{ED}_{50}$ from $10.0 \pm 2.5$ to $6.9 \pm 2.5 \mu \mathrm{g} / \mathrm{kg} / \mathrm{min}(p=0.006)$. The BHR was completely abolished in both Groups ID and IE, with no changes in $\mathrm{ED}_{50}(9.5 \pm 3.6$ vs. $10.7 \pm 4.7, \mathrm{p}=0.33$ and $10.6 \pm 2.1$ vs. $9.8 \pm 3.5 \mu \mathrm{g} / \mathrm{kg} / \mathrm{min} p=0.56$, respectively).

Conclusions: These findings suggest that a LVD following coronary ischaemia consistently induces BHR. The more consistent efficacy of both treatment strategies in preventing BHR than in treating the adverse pulmonary vascular consequences suggests the benefit of both calcium channel blockade and ACE inhibition to counteract the airway susceptibility following a LVD.
\end{abstract}

\section{Background}

The results of previous clinical and experimental studies clearly established that a left ventricular dysfunction (LVD) leads to a lung function impairment manifested in airflow limitation and compromised lung compliance $[1,2]$. There is also increasing evidence that the diminished airway function following a LVD results in the development of bronchial hyperreactivity (BHR) in response to exogenous constrictor stimuli [1,3-5]. The pulmonary congestion subsequent to chronic LVD in

\footnotetext{
* Correspondence: petak@dmi.szote.u-szeged.hu

'Department of Medical Physics and Informatics, University of Szeged, 9

Koranyi fasor, H-6720, Szeged, Hungary

Full list of author information is available at the end of the article
}

patients advances the development of clinical symptoms, such as wheezing, coughing, dyspnea and recurrent bronchospasm triggered by exposures to various provocation agonists $[1,4]$. A number of factors may contribute to the development of BHR following a LVD, including a decrease in airway cross-sectional area [6] due to the compression of the airways by the dilated pulmonary vessels [4], an elevated capillary hydrostatic pressure leading to mucosal swelling $[1,3]$, and airway wall hypertrophy [7].

In clinical practice, different treatment strategies are considered in the presence of LVD in order to improve the cardiac output, to advance fluid clearance and to decrease pulmonary congestion. Angiotensin converting 
enzyme (ACE) inhibitors are commonly regarded as first-line therapy through which to counteract the reninangiotensin pathway and hence the production and secretion of aldosterone [8], with an ultimate reduction of the systemic vascular resistance and relief of the vascular engorgement. Alternatively, there has been some interest in the blockade of calcium entry, which can potentially improve the left ventricular function via systemic arterial vasodilation, leading to a reduced ventricular afterload, reflex activation of the sympathetic nervous system and direct improvement of the myocardial inotropic depression $[9,10]$. Despite these well-established beneficial effects of these treatments on the haemodynamic outcomes, there have been no studies aimed at establishing how these treatment strategies ultimately alter the adverse pulmonary consequences of a LVD. Accordingly, the effectiveness of such treatments as concerns the alterations in the basal airway and tissue mechanical properties, lung volume and airway responsiveness has not been characterized.

We therefore set out to explore the pulmonary consequences of these common treatment strategies, applied in the presence of a sustained elevation in pulmonary venous pressure following the induction of a LVD in a well-established experimental model [11] mimicking the adverse pulmonary symptoms of chronic lung congestion. Changes in pulmonary haemodynamics, basal airway and tissue mechanics and lung responsiveness were characterized following a decrease in the insult from the vascular remodelling by i) an ACE inhibitor combined with a diuretic and ii) a calcium channel blocker.

\section{Methods}

\section{Animal preparations}

The experimental protocol was approved by the Experimental Ethics Committee of the University of Geneva (No. 09-45) and the Animal Welfare Committee of the Canton of Geneva (No. 1051/3542/3). On the experimental days, adult male Sprague-Dawley rats (initially weighing 342-395 g) were anaesthetized by the intraperitoneal injection of chloral hydrate $(350 \mathrm{mg} / \mathrm{kg})$. Tracheal intubation was achieved with a polyethylene cannula (16-gauge, Braun, Melsungen, Germany) and the rats were allowed to breathe spontaneously for the lung volume measurement. The rats were then mechanically ventilated with a tidal volume of $7 \mathrm{ml} / \mathrm{kg}$ body weight, a positive end-expiratory pressure of $2.5 \mathrm{cmH}_{2} \mathrm{O}$, and a respiratory rate of $60-70 / \mathrm{min}$ (constant volume-cycled rodent ventilator, model 683, Harvard Apparatus Co Inc., South Natick, MA, USA). After the completion of plethysmography, the femoral vein was cannulated and a continuous iv infusion of midazolam $(1 \mathrm{mg} / \mathrm{kg} / \mathrm{h})$ with fentanyl $(40 \mu \mathrm{g} / \mathrm{kg} / \mathrm{h})$ and atracurium $(1 \mathrm{mg} / \mathrm{kg} / \mathrm{h})$ was administered to ensure an adequate anaesthesia and analgesia level throughout the study. The femoral artery was cannulated (Abocath $22 \mathrm{G}$ ) and attached to a pressure transducer (Model 156 PCE 06-GW2, Honeywell, Zürich, Switzerland) for continuous blood pressure monitoring. An arterial line was also used for blood gas analysis (Model 505, Acid Base Laboratory, Copenhagen, Denmark). The airway pressure, arterial pressure, ECG and rectal temperature were continuously monitored by a data collection and acquisition system (Biopac, Santa Barbara, CA, USA).

\section{Surgical preparations for the induction of LVD}

An additional bolus of fentanyl was administered intraarterially $(15 \mu \mathrm{g} / \mathrm{kg})$ to strengthen the level of analgesia. The heart in each rat was then surgically exposed by a left lateral thoracotomy ( $5^{\text {th }}$ intercostal). A prophylactic dose of lidocaine ( $2 \mathrm{mg} / \mathrm{kg}$ intra-arterially) was administered to prevent arrhythmias that may arise from ligation of the coronary artery. After opening of the pericardium, a silk thread was sutured around the proximal part of the descending interventricular coronary artery (next to the departure of the pulmonary artery).

\section{Treatments and protocol groups}

In the animals assigned to Groups I $(n=10)$, ID $(n=9)$ and IE $(n=10)$, the knot was tightened to induce a LVD via myocardial ischaemia, while in the rats enrolled in Group C $(n=10)$, sham surgery was performed by removal of the silk thread without occlusion of the coronary artery. The thorax was closed after removing the residual air in the pleural cavity, and a long-acting local anaesthetic (bupivacaïne $0.25 \%, 0.8-1.2 \mathrm{ml}$ ) was infiltrated around the surgical wounds. $0.5 \mathrm{mg} / \mathrm{kg}$ of buprenorphine $\left(\right.$ Temgesic $^{\circledR}$ ) was injected subcutaneously before the rat was allowed to recover from the anaesthesia, and this dose was repeated $18 \mathrm{~h}$ later to ensure adequate analgesia.

After creation of the LVD, the animals were randomized to be included to receive no further treatment during the 8-week study period (Group I), regular treatment with a calcium channel blocker (diltiazem $10 \mathrm{mg} /$ $\mathrm{kg}$ /day via daily ip injection) for 8 weeks (Group ID), or regular treatment with an ACE inhibitor (enalapril, $10 \mathrm{mg} / \mathrm{kg} /$ day, via oral gavage) supplemented with a diuretic (furosemide $2 \mathrm{mg} / \mathrm{kg} /$ day, via oral gavage) until the next experimental day 8 weeks later (Group IE). The daily itraperitoneal and oral treatments were performed by gentle restraining of the animals without sedation. The route of administration of the treatment agents and the doses were chosen on the basis of their pharmacokinetic properties and the manufacturers' recommendations. Eight weeks after the completion of the first part of the investigations, the animals were re-evaluated as detailed above, and the second part of the investigations 
was performed. The scheme of the experiments is presented in Figure 1.

\section{Measurements of end-expiratory lung volume (EELV)}

After the anaesthesia with chloral hydrate and the surgical preparations, the measurements of EELV were performed in all groups by using a body plethysmograph, as detailed earlier [12]. Briefly, the trachea was occluded at end-expiration until 3 or 4 spontaneous inspiratory efforts had been generated by the animal in the closed box. The changes in tracheal pressure and plethysmograph box pressure during these manoeuvres were recorded, and EELV was calculated by applying Boyle's law to the relationship between the tracheal pressure and the box pressure after correction for the box impedance [13]. The recordings of box pressure were corrected for the thermal characteristics of the plethysmograph [13]; this minimized the biasing effects of the different breathing frequencies during the inspiratory efforts. The sufficient spontaneous breathing manoeuvres were required for the measurement of EELV were not always achieved in the anaesthetized animals: these measurements were successfully accomplished in 8, 7, 9 and 10 animals in Groups C, I, ID and IE, respectively.

\section{Forced oscillatory measurements}

The contributions of the airway and tissue mechanical properties to the total respiratory resistance were assessed by the forced oscillation technique in the anesthetized animals through measurement of the mechanical impedance of the respiratory system (Zrs), as described in detail previously [14,15]. Briefly, the tracheal cannula was connected from the respirator to a loudspeaker-in-box system at end-expiration. Zrs was measured with the wave-tube technique by introducing pseudorandom forced oscillations at frequencies between 0.5 and $21 \mathrm{~Hz}$ at end-expiration. Two identical pressure transducers (Model 33NA002D, ICSensors, Malpitas, CA, USA) were used to measure the lateral pressures at the loudspeaker and at the tracheal end of the wave-tube, and Zrs was calculated as the load impedance of the wave-tube by using fast Fourier transformation [14].

To separate the airway and tissue mechanics, a model containing a frequency-independent resistance (Raw) and inertance (Iaw) in series with a constant-phase tissue model [16] including tissue damping (G) and elastance $(\mathrm{H})$ was fitted to the Zrs spectra by minimizing the differences between the measured and modelled impedance values:

$$
\mathrm{Zrs}=\mathrm{Raw}+j \omega \mathrm{Iaw}+(G-\mathrm{jH}) / \omega^{\alpha}
$$

where $j$ is the imaginary unit, $\omega$ is the angular frequency $(2 \pi f)$ and $\alpha=2 / \pi \arctan (H / G)$. When this model is fitted to Zrs spectra, the parameter Raw is primarily related to the overall airway geometry, as the contribution of the chest wall to the frequency-independent Newtonian resistance is minor [17]. Similarly, the inertia of the gas in the airways predominates in the parameter Iaw [17]. The equivalent dose causing a $50 \%$ increase in Raw $\left(E_{50}\right)$ was calculated in each rat by linear interpolation. Following these measurements, pulmonary arterial pressure was measured as described below. The specific airway resistance (SRaw) was calculated as Raw*EELV.

\section{Measurement of pulmonary haemodynamics}

The pulmonary arterial pressure was measured by introducing a small catheter (polyethylene tubing, ID $0.88 \mathrm{~mm}$, Portex, Hythe, GB) into the pulmonary artery via the jugular vein before the animals were sacrificed. Since the diastolic pulmonary arterial pressure $\left(\mathrm{Pap}_{\mathrm{D}}\right)$ reflects the left atrial pressure and thus the end-diastolic left ventricular pressure, we used this parameter to express the haemodynamic consequence of a LVD. Because of the technical demands of this manoeuvre for successful introduction of the miniature catheter in rats, this measurement proved technically acceptable only in subgroups of the main study groups, involving 7, 6, 7 and 10 rats in Groups C, I, ID and IE, respectively.

\section{Weeks}

\begin{tabular}{|c|c|c|c|c|c|}
\hline Group & Surge & 0 & 4 & 6 & 8 \\
\hline C & SHAM & \multirow{4}{*}{ 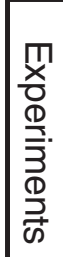 } & \multicolumn{2}{|c|}{ No treatment } & \multirow{4}{*}{ 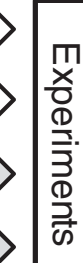 } \\
\hline I & LVD & & No tre & & \\
\hline ID & LVD & & Daily diltiazem $10 \mathrm{mg} / \mathrm{kg}$ & traperitoneal pump & \\
\hline IE & LVD & & Daily enalapril $10 \mathrm{mg} / \mathrm{kg}$ & mide $2 \mathrm{mg} / \mathrm{kg}$ orally & \\
\hline
\end{tabular}

Figure 1 Scheme of the experimental protocol. 


\section{Measurement protocol and bronchoprovocation challenges}

The experimental protocol included the follow-up of the same animals through the 8-week study period by performing the identical procedure at weeks 0 and 8 (Figure 1). On each occasion, the rats were anaesthetized and prepared as detailed above. EELV was first measured while the rats were breathing spontaneously. A femoral vein was then cannulated for drug delivery. When stable haemodynamic and respiratory mechanical conditions had been reached, we recorded a set of Zrs data including the collection of 4-6 6-s data epochs under the baseline conditions. Increasing doses of iv methacholine (MCh) were then administered at doses of 2,6 and $18 \mu \mathrm{g} / \mathrm{kg} /$ min. 3-5 Zrs recordings were made at each infusion rate during the steady-state bronchoconstriction that developed, usually 3-5 min after the onset of MCh. Following these measurements, $\mathrm{Pap}_{\mathrm{D}}$ was measured as described above.

\section{Statistical analyses}

The scatters in the parameters were expressed by the SD values. The Kolmogorov-Smirnov test was used to check data for normality. Two-way repeated measures analysis of variances (ANOVA), with variables of treatment (i.e. the protocol groups) and measurement condition (i.e. weeks 0 and 8), was used to evaluate the effects of the various types of medication on the respiratory mechanical parameters and EELV and the alterations in lung responsiveness to the constrictor challenge. The pulmonary haemodynamic differences between the groups were tested by using oneway ANOVA. Pairwise comparisons were performed by means of Student-Newman-Keuls multiple comparison procedures. The Pearson correlation test was used to assess the strength of associations between variables. Statistical tests were carried out with the significance level set at $\mathrm{p}<0.05$.

\section{Results}

There was no significant difference in body weight between the protocol groups at the beginning of the study (ranging from $308 \pm 56 \mathrm{~g}$ to $314 \pm 62 \mathrm{~g}, \mathrm{p}=0.71$ ). Eight weeks later, however, the body weight of the animals in Group IE (463 $\pm 9 \mathrm{~g})$ was significantly lower than those of the rats in Groups C $(518 \pm 27 \mathrm{~g}, \mathrm{p}<0.001)$, I $(498 \pm$ $32 \mathrm{~g}, \mathrm{p}=0.014)$ or ID $(498 \pm 30 \mathrm{~g}, \mathrm{p}=0.014)$. The changes in the breathing frequency during EELV measurements reflected these developmental changes with decreases from $66 \pm 22,74 \pm 13,78 \pm 20$ and $74 \pm 16$ breath/min at week $0(\mathrm{p}=0.6)$ to $39 \pm 8,44 \pm 14,43 \pm 18$ and $54 \pm 23 \mathrm{breath} / \mathrm{min}$ at week $8(\mathrm{p}=0.29)$ in Groups C, I, ID and IE, respectively.

The baseline values of the airway and respiratory tissue mechanical parameters exhibited significant decreases ( $p<0.001$ for all) between the two study occasions (Figure 2).
We found that none of the parameters displayed a significant difference between the study groups under the baseline condition ( $\mathrm{p}=0.16,0.87$ and 0.38 for Raw, $\mathrm{G}$ and $\mathrm{H}$, respectively). The lack of any significant interaction between the measurement time and the protocol group demonstrated that the induction of the LVD and its treatments had no significant effects on the changes in the mechanical parameters ( $\mathrm{p}=0.52,0.25$ and 0.54 for Raw, $\mathrm{G}$ and $\mathrm{H}$, respectively).

EELV and the baseline SRaw increased significantly ( $p<0.001$ for both) in all groups by the end of the 8-week study period (Figure 3 ). The percentage relative increase in EELV after 8 weeks was significantly higher in the Group ID $(118 \pm 48 \%)$ thank in Group C $(75 \pm 30 \%, p=0.048)$, and there was a tendency for an excessive EELV increase in group I $(145 \pm 107 \%, p=0.09)$. Mild but statistically significantly more pronounced elevation in SRaw were observed in the rats of Group IE ( $\mathrm{p}=0.047)$ by the end of the 8-week study period (Figure 3).

$\mathrm{MCh}$ provocations induced a dose-dependent bronchoconstriction in all groups $(\mathrm{p}<0.001$, Figure 4$)$. No change in airway responsiveness was detected in Group $C(p=0.7)$, whereas the induction of a LVD led to the development of BHR in the rats in Group I ( $=0.02)$, as illustrated by the significant upward shift in the dose-response curve. Each of the treatment strategies exerted a protective effect against this hyperresponsiveness to $\mathrm{MCh}$, with abolition of the difference in airway responsiveness in Groups ID ( $p=0.3$ week 0 vs. week 8$)$ and IE ( $\mathrm{p}=0.65$ week 0 vs. week 8$)$. These findings were reflected in the lack of significant change in $\mathrm{ED}_{50}$ in Groups $\mathrm{C}(9.2 \pm 1.9$ vs. $10.2 \pm 4.4 \mu \mathrm{g} / \mathrm{kg} / \mathrm{min}, \mathrm{p}=$ 0.65), ID (9.5 \pm 3.6 vs. $10.7 \pm 4.7 \mu \mathrm{g} / \mathrm{kg} / \mathrm{min}, \mathrm{p}=0.33)$ and IE $(10.6 \pm 2.1$ vs. $9.8 \pm 3.5 \mu \mathrm{g} / \mathrm{kg} / \mathrm{min}, \mathrm{p}=0.56)$, whereas it decreased markedly in the rats in Group I $(10.0 \pm 2.5$ vs. 6.9 $\pm 2.5 \mu \mathrm{g} / \mathrm{kg} / \mathrm{min}, \mathrm{p}=0.006$ ).

The induction of the LVD increased $\mathrm{Ppa}_{\mathrm{D}}$ in all groups where pulmonary hypertension was generated by coronary ischaemia (Figure 5), with variable but significant elevations in Groups I $(\mathrm{p}=0.004)$, ID $(\mathrm{p}=0.013)$ and IE $(\mathrm{p}=0.006)$.

The analyses of the associations between the changes in pulmonary haemodynamics and respiratory mechanics revealed no significant correlations between the magnitude of pulmonary haemodynamic changes expressed as $\operatorname{Pap}_{\mathrm{D}}$ and the alterations in lung responsiveness expressed as the difference in $\mathrm{ED}_{50}$ between weeks 0 and 8 $(\mathrm{R}=0.097, \mathrm{p}=0.61$, Figure 6). Furthermore, no significant correlation was observed between $\mathrm{Pap}_{\mathrm{D}}$ and the 8-week changes in $\operatorname{EELV}(\mathrm{R}=0.033, \mathrm{p}=0.86)$, while there was a tendency to a strong association between $\operatorname{Pap}_{\mathrm{D}}$ and the 8week changes in Raw $(R=-0.35, p=0.06)$.

\section{Discussion}

Various treatment strategies are applied in the presence of a LVD in order to enhance the cardiac output 

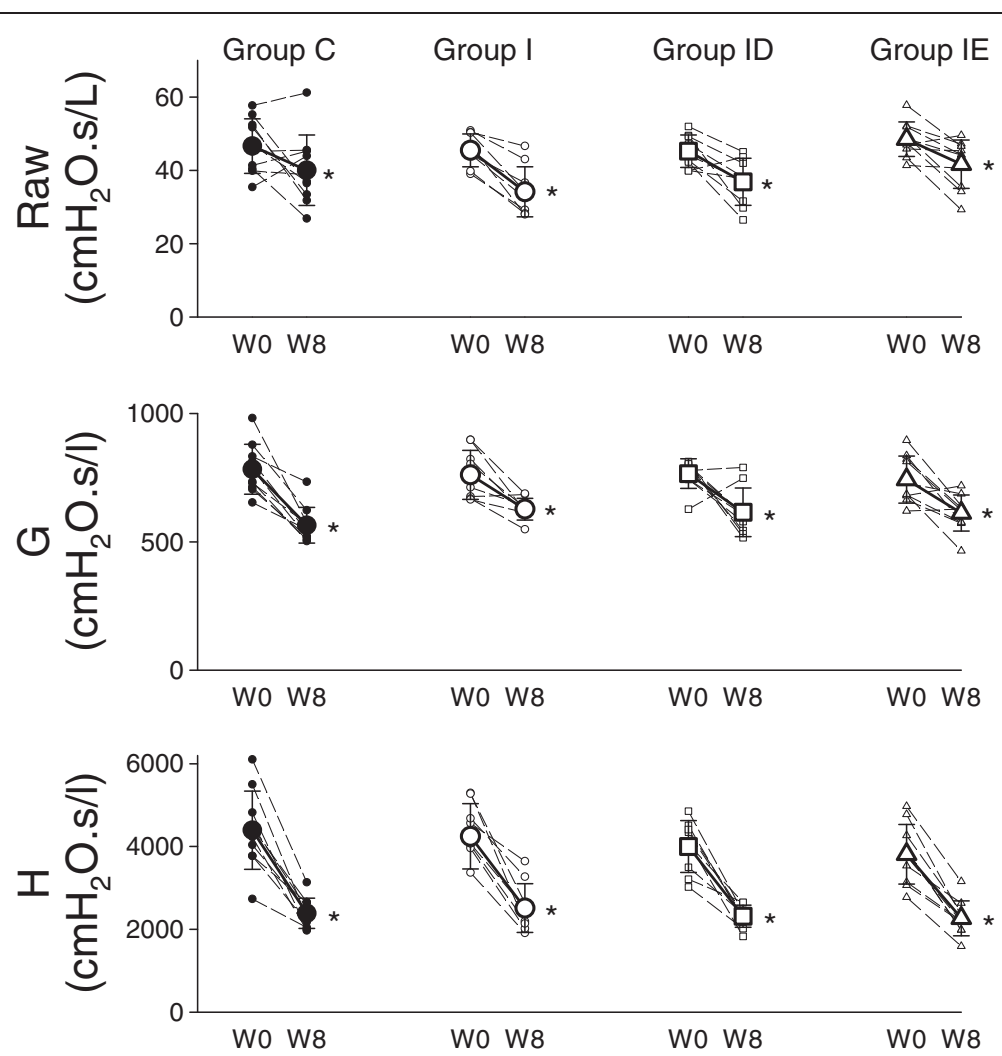

Figure 2 Changes in the baseline values of the respiratory mechanical parameters obtained by forced oscillation before (W0) and 8 weeks after the induction of a LVD (W8) in rats that underwent sham surgery (Group C), and in rats with a LVD without subsequent treatment (Group I) or supplemented with treatment with calcium channel (Group ID) or ACE blockers (Group IE). Raw: airway resistance, G: tissue damping, H: tissue elastance. Thin dashed lines with small symbols: individual animals; thick lines with large symbols: group means. *: $p<0.05$ W0 vs. W8.
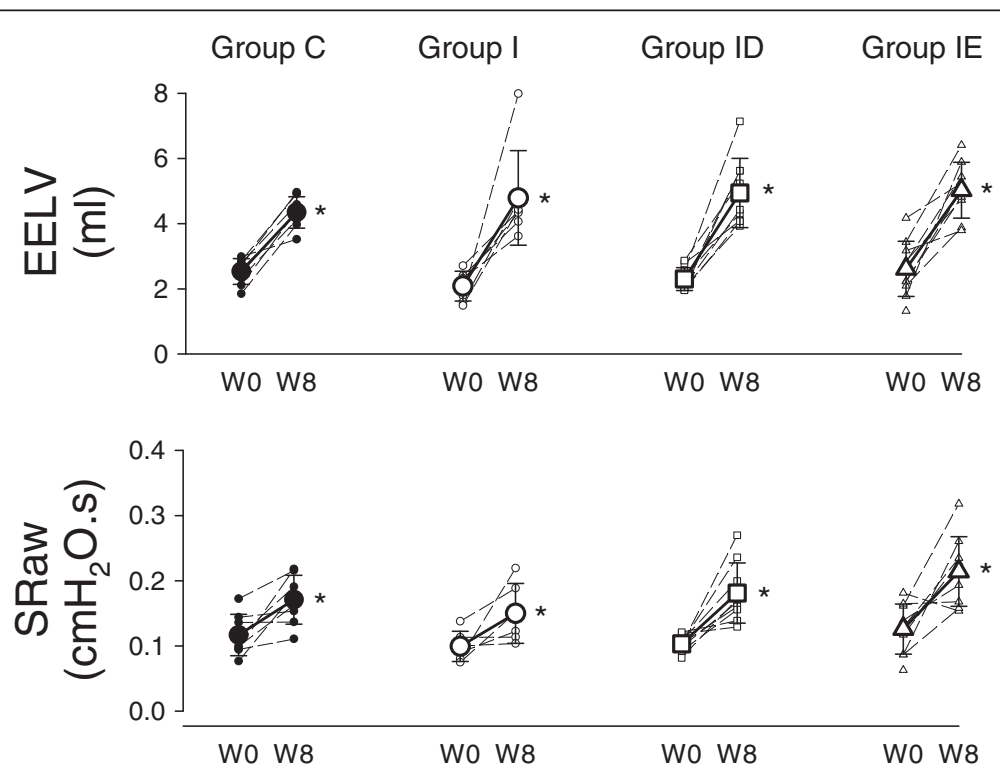

Figure 3 Changes in the end-expiratory lung volume (EELV), its value normalized to body weight (Normalized EELV) and the specific airway resistance (SRaw) before (W0) and 8 weeks after LVD induction (W8) in rats that underwent sham surgery (Group C), and in rats with a LVD without subsequent treatment (Group I) or supplemented with treatment with calcium channel (Group ID) or ACE blockers (Group IE). Thin dashed lines with small symbols: individual animals; thick lines with large symbols: group means. *: $p<0.05$ W0 vs. W8. 


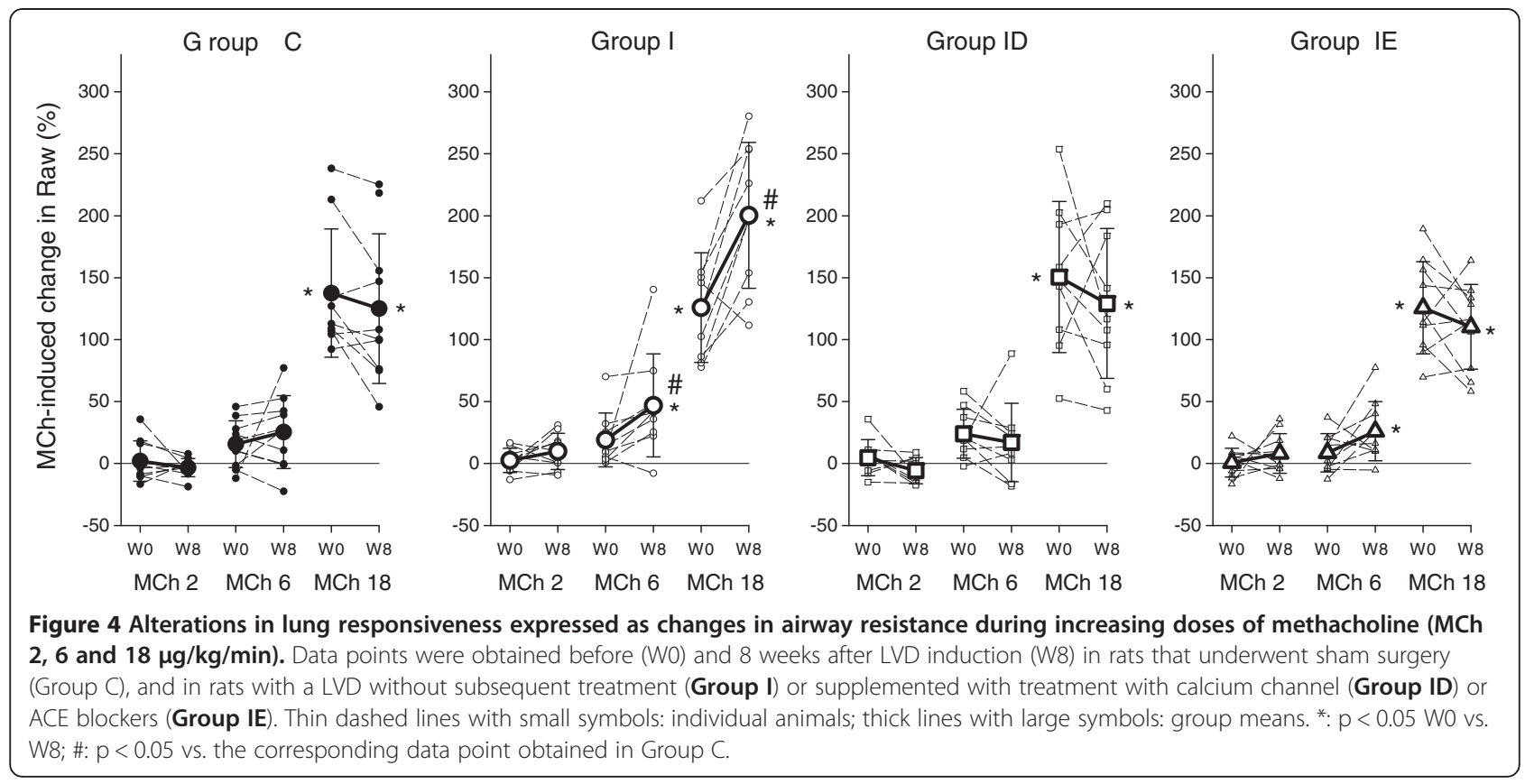

by optimizing the preload of the left ventricle and subsequently decreasing the pulmonary vascular engorgement or by decreasing the systemic vascular resistance. In the present study, we investigated the abilities of treatments considered against a LVD to protect the potentially adverse pulmonary consequences of left

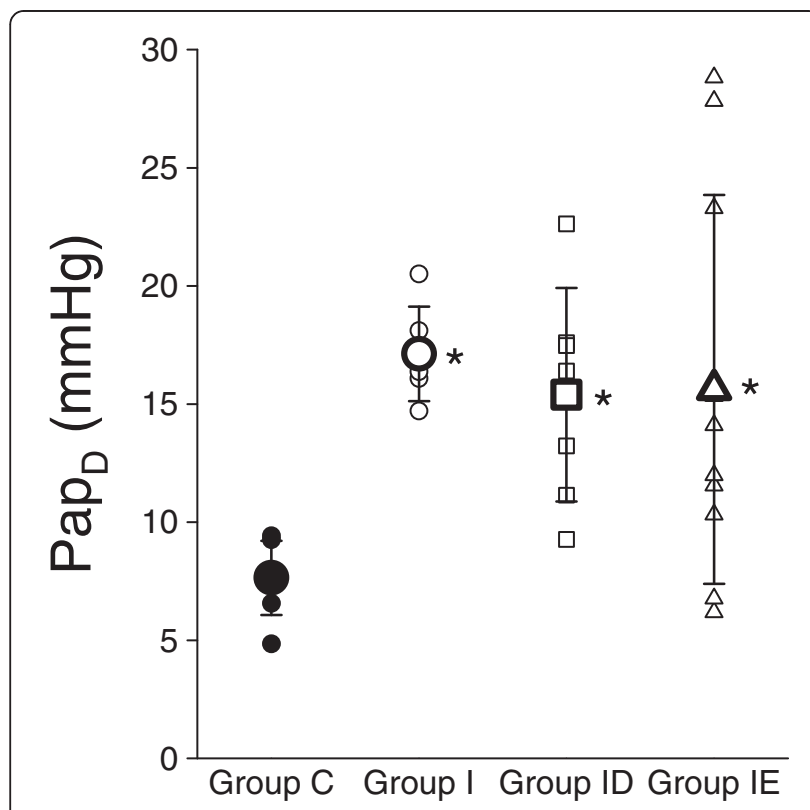

Figure 5 Diastolic pulmonary arterial pressures in the rats involved in the different groups. Group C: control group that underwent sham surgery, Group I: rats with a LVD without further treatment, Group ID: rats with a LVD treated with a calcium channel blocker (diltiazem). Group IE: rats with a LVD treated with an ACE blocker (enelapril). *: $p<0.05$ vs. the data obtained in Group C. heart failure. We applied a well-validated animal model of coronary ischaemia to induce a sustained elevation of left atrial pressure, leading to postcapillary PHT and the subsequent development of BHR. Under this pathophysiological condition, both treatment strategies (based on blockade either of the rennin-angiotensin pathway supplemented with diuretics or of the calcium channels) abolished the enhanced lung responsiveness to exogenous constrictor stimuli without affecting the lung volume increase. Examination of the relationships between the pulmonary haemodynamic and lung functional changes revealed that the diastolic Pap level and the magnitude of the lung responsiveness were dissociated.

In agreement with the earlier reported findings $[11,18]$, the induction of left ventricular myocardial ischaemia led consistently to postcapillary PHT, as proved by the marked elevations in $\operatorname{Pap}_{\mathrm{D}}$ in the present study. The achievement of more detailed experimentation to characterize the altered pulmonary haemodynamics in the present study was complicated by the small size of the model animal and the lack of a catheter-tip balloon facilitating its introduction into the pulmonary artery. This technical drawback was the main reason for the availability of a technically acceptable $\mathrm{Pap}_{\mathrm{D}}$ recording in only about two-thirds of the rats, which agrees well with the previous success rate in this species when a similar technique was used $[11,19]$. Due to the technically challenging nature of the surgical preparation of LVD, one control group of rats with LVD was assessed (Group I) instead of involving separate control groups of rats with ip saline injections or oral placebo treatment. Since the 


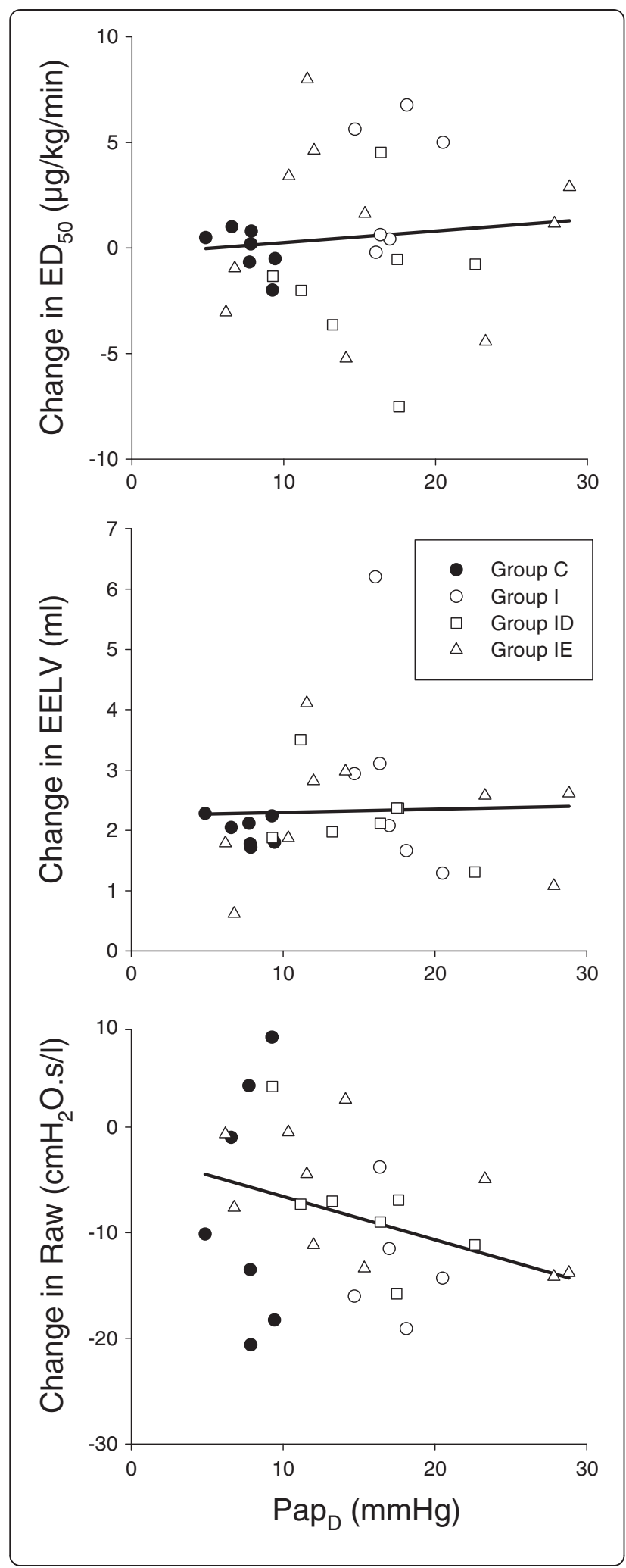

Figure 6 Relationships between the diastolic pulmonary arterial pressures $\left(\mathrm{Ppa}_{\mathrm{D}}\right)$ measured 8 weeks after LVD induction and the changes in lung responsiveness to $M C h\left(E_{50}\right)$ in the end-expiratory lung volume (EELV) and in the airway resistance (Raw). Data points were obtained in rats that underwent sham surgery (Group C), and in rats with a LVD without subsequent treatment (Group I) or supplemented with treatment with calcium channel (Group ID) or ACE blockers (Group IE).

ip (Group ID) and oral (Group IE) treatments of LVD necessitated only mild restrains of the animals for short period of times, the interventions related to the treatments per se were not likely to bias our findings.

In the presence of a LVD, the systematic enhancement of the airway responsiveness to exogenous constrictor stimuli as a hallmark feature of BHR was confirmed in the present study [11]. In complete agreement with a previous observation [20], there was no significant correlation between the degree of lung congestion estimated from $\mathrm{Pap}_{\mathrm{D}}$ and the severity of BHR (Figure 6), confirming that a more severe pulmonary vascular congestion after a LVD does not necessarily involve a more severe BHR. This consistent finding demonstrates that the enhanced $\mathrm{MCh}$ responsiveness following a sustained LVD is not directly related to the altered geometry of the congested pulmonary vessels. Rather, LVD may trigger a cascade mechanism via a release of numerous broncho- and vasoactive mediators, causing the development of vascular remodelling and subsequent BHR.

It is noteworthy that BHR was observed previously on use of a constrictor challenge with serotonin [11] and this feature was fully reproduced in the present study by applying $\mathrm{MCh}$ as a provocation agonist. Since serotonin and $\mathrm{MCh}$ induce bronchoconstriction by activating different receptors, with the former acting on the muscarinic and the latter stimulating the 5-hydroxytryptamine receptors, the consistent detection of BHR demonstrates that, in the presence of a sustained LVD, the enhanced lung responsiveness is independent of the receptor subtype via which the different agonists exert their bronchoconstrictive potentials.

While all respiratory mechanical parameters at the baseline level exhibited marked decreases in the 8-week study period, due to the somatic growth (Figure 2), neither the presence of the LVD nor the treatments affected them significantly. This finding agrees with the previous experimental data indicative of a lack of change in the baseline values of airway or respiratory tissue mechanics following a sustained pre- $[19,21]$ or postcapillary PHT [11]. Furthermore, this result is in line with previous clinical observations that demonstrated no significant effects of a LVD on the basal airway [22] or respiratory [23] resistance. The present study is the first to demonstrate a follow-up in the lung volume changes before and after a sustained LVD. The changes observed in EELV and 
in the breathing rate during the 8-week study period can be explained almost fully by the lung growth (Figure 3, top). Since the EELV values were corrected for the thermal characteristics of the plethysmograph, the uniform alterations in the breathing rate in the protocol groups was not likely to bias our estimates [13]. The strong tendency for excessive increases in the EELV in the rats with a LVD may be attributed to the involvement of the autonomous nervous system in the presence of hypoxia [12,24] that might have been present after the induction of LVD [1].

The use of calcium channel blockers in the presence of LVD has been challenged in clinical practice in the past decade [25]. However, among the calcium antagonists, diltiazem was demonstrated to exhibit the best profile without a significant negative inotropic effect and an improvement in cardiac function following coronary ischaemia [26]. Our findings suggest that diltiazem did not inhibit the pulmonary vascular engorgement, as revealed by the preservation of a high $\mathrm{Pap}_{\mathrm{D}}$ in the majority of the rats treated for 8 weeks (Figure 5). Conversely, diltiazem prevented the development of BHR (Figure 4) by diminishing the bronchoconstriction at all doses of MCh. This beneficial effect is most probably due to the effect of diltiazem on the calcium channels, with a consequent decrease in the $\mathrm{Ca}^{2+}$ influx into the smooth muscle cells. Since $\mathrm{Ca}^{2+}$ is essential for the contractility of the airway smooth muscle cell, diltiazem may exert its potential protection effect via this cellular mechanism [27].

A LVD following coronary ischaemia leads to a decrease in cardiac output and stimulates the renin-angiotensinaldosterone pathway, which then results in renal sodium and water retention, contributing further to the overload, and hence increasing the preload of the left ventricle $[28,29]$. A combination of enalapril and diuretics is therefore the first-line treatment of cardiac failure, through inhibition of this mechanism, leading to optimization of the preload of the left ventricle and a subsequent decrease in the pulmonary venous pressure. In agreement with the literature, the 8-week treatment with enalapril slightly retarded the body weight gain most probably by affecting the metabolic homeostasis [30]. Furthermore, this treatment proved to be beneficial in the majority of animals, with a decrease in $\mathrm{Pap}_{\mathrm{D}}$, though some rats still exhibited a very high pulmonary venous pressure (Figure 5). Despite this fairly diverse pulmonary vascular effect, enalapril exhibited a uniform beneficial effect on the respiratory mechanics and airway responsiveness. It is noteworthy that this treatment slightly but statistically significantly elevated the baseline value of SRaw, which may be related to the irritating properties of enalapril on the airways, occasionally causing mild airway obstruction [31]. As regards the potential of $\mathrm{ACE}$ inhibition to affect BHR, there have been conflicting findings, with some authors promoting its beneficial profile [32,33], others reporting no change in lung reactivity [34] and some even demonstrating a worsening airway responsiveness [35,36]. Our results are in line with those suggesting that ACE inhibition with enalapril may counterbalance the constrictor effect of angiotensin on the airway smooth muscle and may blunt the BHR observed following cholinergic stimulation with MCh [32]. Since the level of angiotensin is expected to increase following a LVD, the blockade of this pathway may be responsible for this beneficial pulmonary profile observed in the present study. Besides this potential beneficial profile of enalapril, the supplemental administration of furosemide may further contribute to the inhibition of BHR, since this diuretics was proved to decrease airway responsiveness in humans [37] and in rodents [38]. This effect may be related to ease of the mechanical load exerted by the congested pulmonary capillaries on the airways and/or to the modulation of the neurotransmission [37].

\section{Summary and conclusions}

Surgical induction of coronary ischaemia in the present study confirmed that a sustained increase in left atrial pressure and subsequently in the pulmonary vasculature enhances the airway responsiveness to exogenous constrictor stimuli and suggested an elevation of static lung volume. The pathogenesis of this phenomenon was effectively prevented by blocking the calcium channels following the insult and by treating the adverse neurohumoral consequences of the LVD by counteracting the adverse vascular effect of the angiotensin pathway. Relating the efficiency of these treatments on the lung responsiveness and the pulmonary haemodynamics revealed that both strategies exhibit more consistent benefit against airway hyperresponsiveness due to pulmonary vascular engorgement than on the rather variable profile on the lung vasculature. The lack of a close association between the severity of BHR and the degree of lung congestion suggests that broncho- and vasoactive mediators were involved rather than alterations in the geometry of the congested pulmonary vessels. The abolition of airway symptoms in patients with a LVD following these treatment strategies is therefore anticipated.

\section{Competing interests}

The authors declare that they have no competing interests.

\section{Authors' contributions}

FP coordinated the various experimental approaches, and contributed to their design and to the manuscript preparation. GA and EL carried out the experiments and the preliminary data analyses. MB participated in the design of the study and in the interpretation of the experimental findings. $\mathrm{WH}$ conducted the design of the study and had a major role in drafting the manuscript. All authors have read and approved the final manuscript.

\section{Acknowledgements}

Supported by Hungarian Basic Scientific Research Grant OTKA K81179 and Swiss National Science Foundation Grant SNSF 32003B-118231. Ferenc Petak is supported by a Bolyai Janos Research Fellowship. 
The authors are grateful for the excellent technical assistance of Jorge-Costa Manuel.

\section{Author details}

'Department of Medical Physics and Informatics, University of Szeged, 9 Koranyi fasor, $\mathrm{H}-6720$, Szeged, Hungary. ${ }^{2}$ Anaesthesiological Investigations Unit, University Hospitals of Geneva, 1 Rue Michel Servet, CH-1205, Geneva, Switzerland. ${ }^{3}$ Paediatric Cardiology Unit, Department of Paediatrics, Geneva Children's Hospital, 6, Rue Willy Donze, CH-1205, Geneva, Switzerland. ${ }^{4}$ Paediatric Anaesthesia Unit, Geneva Children's Hospital, University Hospitals of Geneva, 6, Rue Willy Donze, CH-1205, Geneva, Switzerland.

Received: 2 August 2012 Accepted: 5 December 2012 Published: 13 December 2012

\section{References}

1. Snashall PD, Chung KF: Airway obstruction and bronchial hyperresponsiveness in left ventricular failure and mitral stenosis. Am Rev Respir Dis 1991, 144:945-956.

2. Gehlbach BK, Geppert E: The pulmonary manifestations of left heart failure. Chest 2004, 125:669-682.

3. Albu G, Peták F, Fontao F, Biton C, Pache JC, Habre W: Mechanisms of airway hyper-responsiveness after coronary ischemia. Respir Physiol Neurobiol 2008, 162:176-183.

4. Cabanes LR, Weber SN, Matran R, Regnard J, Richard MO, Degeorges ME, Lockhart A: Bronchial hyperresponsiveness to methacholine in patients with impaired left ventricular function. N Engl J Med 1989, 320:1317-1322.

5. Rolla G, Bucca C, Caria E, Scappaticci E, Baldi S: Bronchial responsiveness in patients with mitral valve disease. Eur Respir J 1990, 3:127-131.

6. Wetzel RC, Herold CJ, Zerhouni EA, Robotham JL: Intravascular volume loading reversibly decreases airway cross-sectional area. Chest 1993, 103:865-870.

7. Haworth SG, Hall SM, Panja M: Peripheral pulmonary vascular and airway abnormalities in adolescents with rheumatic mitral stenosis. Int J Cardio 1988, 18:405-416.

8. Chatterjee $\mathrm{K}$ : Congestive heart failure: what should be the initial therapy and why? Am J Cardiovasc Drugs 2002, 2:1-6.

9. Henry PD: Comparative pharmacology of calcium antagonists: nifedipine, verapamil and diltiazem. Am J Cardiol 1980, 46:1047-1058.

10. Zema MJ, Perlmutter S, Mankes S, Nikitopoulos C: Diltiazem treatment for the management of ischaemia in patients with poor left ventricular function: safety of long term administration. Br Heart J 1987, 58:512-517.

11. Albu G, Petak F, Fontao F, Biton C, Pache JC, Habre W: Mechanisms of airway hyper-responsiveness after coronary ischemia. Respir Physiol Neurobiol 2008, 162:176-183.

12. Habre W, Janosi TZ, Fontao F, Meyers C, Albu G, Pache JC, Petak F: Mechanisms for lung function impairment and airway hyperresponsiveness following chronic hypoxia in rats. Am J Physiol Lung Cell Mol Physiol 2010, 298:L607-L614.

13. Janosi TZ, Adamicza A, Zosky GR, Asztalos T, Sly PD, Hantos Z Plethysmographic estimation of thoracic gas volume in apneic mice. J Appl Physiol 2006, 101:454-459.

14. Petak F, Hantos Z, Adamicza A, Asztalos T, Sly PD: Methacholine-induced bronchoconstriction in rats: effects of intravenous vs. aerosol delivery. J Appl Physiol 1997, 82:1479-1487.

15. Petak F, Habre W, Hantos Z, Sly PD, Morel DR: Effects of pulmonary vascular pressures and flow on airway and parenchymal mechanics in isolated rat lungs. J Appl Physiol 2002, 92:169-178.

16. Hantos Z, Daroczy B, Suki B, Nagy S, Fredberg JJ: Input impedance and peripheral inhomogeneity of dog lungs. J App/ Physiol 1992, 72:168-178.

17. Petak F, Hall GL, Sly PD: Repeated measurements of airway and parenchymal mechanics in rats by using low-frequency oscillations. J Appl Physiol 1998, 84:1680-1686.

18. Jasmin JF, Calderone A, Leung TK, Villeneuve L, Dupuis J: Lung structural remodeling and pulmonary hypertension after myocardial infarction: complete reversal with irbesartan. Cardiovasc Res 2003, 58:621-631.

19. Habre W, Albu G, Janosi TZ, Fontao F, von Ungern-Sternberg BS, Beghetti M, Petak F: Prevention of bronchial hyperreactivity in a rat model of precapillary pulmonary hypertension. Respir Res 2011, 12:58.
20. Sasaki F, Ishizaki T, Mifune J, Fujimura M, Nishioka S, Miyabo S: Bronchial hyperresponsiveness in patients with chronic congestive heart failure. Chest 1990, 97:534-538.

21. von Ungern-Sternberg BS, Habre W, Regli A, Pache JC, Fontao F, Janosi TZ, Beghetti M, Petak F: Precapillary pulmonary hypertension leads to reversible bronchial hyperreactivity in rats. Exp Lung Res 2010, 36:129-139.

22. Yap JC, Moore DM, Cleland JG, Pride NB: Effect of supine posture on respiratory mechanics in chronic left ventricular failure. Am J Respir Crit Care Med 2000, 162:1285-1291.

23. Spyratos DG, Glattki GP, Sichletidis LT, Patakas D: Assessment of respiratory mechanics by impulse oscillometry in orthopneic patients with acute left ventricular failure. Heart Lung 2011, 40:97-104.

24. Vizek M, Frydrychova M, Houstek S, Palecek F: The role of vagal nerves in changes of functional residual lung capacity during acute hypoxia. Physiol Bohemoslov 1983, 32:318-323.

25. Boden WE, van Gilst WH, Scheldewaert RG, Starkey IR, Carlier MF, Julian DG, Whitehead A, Bertrand ME, Col JJ, Pedersen OL, et al: Diltiazem in acute myocardial infarction treated with thrombolytic agents: a randomised placebo-controlled trial. Incomplete Infarction Trial of European Research Collaborators Evaluating Prognosis post-Thrombolysis (INTERCEPT). Lancet 2000, 355:1751-1756.

26. Follath $\mathrm{F}$ : The role of calcium antagonists in the treatment of myocardial ischemia. Am Heart J 1989, 118:1093-1096. discussion 1096-1097.

27. Triggle DJ: Calcium, the control of smooth muscle function and bronchial hyperreactivity. Allergy 1983, 38:1-9.

28. Dube P, Weber KT: Congestive heart failure: pathophysiologic consequences of neurohormonal activation and the potential for recovery: part I. Am J Med Sci 2011, 342:348-351.

29. Dube P, Weber KT: Congestive heart failure: pathophysiologic consequences of neurohormonal activation and the potential for recovery: part II. Am J Med Sci 2011, 342:503-506.

30. Santos EL, de Picoli Souza K, da Silva ED, Batista EC, Martins PJ, D'Almeida V, Pesquero JB: Long term treatment with ACE inhibitor enalapril decreases body weight gain and increases life span in rats. Biochem Pharmacol 2009, 78:951-958.

31. Lunde H, Hedner T, Samuelsson O, Lotvall J, Andren L, Lindholm L, Wiholm BE: Dyspnoea, asthma, and bronchospasm in relation to treatment with angiotensin converting enzyme inhibitors. BMJ 1994, 308:18-21.

32. Rubini $A$, Redaelli M, Parmagnani A: The effect of angiotensin-converting enzyme inhibition by captopril on respiratory mechanics in healthy rats J Enzyme Inhib Med Chem 2012, 27(6):854-860.

33. Watanabe K, Myou S, Fujimura M, Tachibana H, Kita T, Nakao S: Importance of the angiotensin type 1 receptor in angiotensin II-induced bronchoconstriction and bronchial hyperresponsiveness in the guinea pig. Exp Lung Res 2004, 30:207-221.

34. Kaufman J, Schmitt S, Barnard J, Busse W: Angiotensin-converting enzyme inhibitors in patients with bronchial responsiveness and asthma. Chest 1992, 101:922-925.

35. Matsubara S, Kikkawa H, Kaminuma O, Ikezawa K: Angiotensin-converting enzyme inhibitors can potentiate ozone-induced airway hyperresponsiveness. Eur J Pharmacol 1997, 337:259-265.

36. Bucknall CE, Neilly JB, Carter R, Stevenson RD, Semple PF: Bronchial hyperreactivity in patients who cough after receiving angiotensin converting enzyme inhibitors. Br Med J (Clin Res Ed) 1988, 296:86-88.

37. Crimi N, Prosperini G, Ciamarra I, Mastruzzo C, Magri S, Polosa R: Changes in neurokinin A (NKA) airway responsiveness with inhaled frusemide in asthma. Thorax 1997, 52:775-779.

38. Wang S, Xiang YY, Ellis R, Wattie J, Feng M, Inman MD, Lu WY: Effects of furosemide on allergic asthmatic responses in mice. Clin Exp Allergy 2011, 41:1456-1467.

doi:10.1186/1465-9921-13-114

Cite this article as: Petak et al:: Prevention of airway

hyperresponsiveness induced by left ventricular dysfunction in rats.

Respiratory Research 2012 13:114. 